\title{
ON RELATIONS BETWEEN WEAK AND STRONG TYPE INEQUALITIES FOR MAXIMAL OPERATORS ON NON-DOUBLING METRIC MEASURE SPACES
}

\author{
DARIUSZ Kosz
}

\begin{abstract}
In this article we characterize all possible cases that may occur in the relations between the sets of $p$ for which weak type $(p, p)$ and strong type $(p, p)$ inequalities for the Hardy-Littlewood maximal operators, both centered and non-centered, hold in the context of general metric measure spaces.
\end{abstract}

2010 Mathematics Subject Classification: Primary: 42B25, 46E30.

Key words: Hardy-Littlewood maximal operators, weak and strong type inequalities, non-doubling metric measure spaces.

\section{Introduction}

Let $\mathbb{X}=(X, \rho, \mu)$ be a metric measure space with a metric $\rho$ and a Borel measure $\mu$ such that the measure of each ball is finite and strictly positive. Define the Hardy-Littlewood maximal operators, centered $M^{c}$ and non-centered $M$, by

$$
M^{c} f(x)=\sup _{r>0} \frac{1}{\mu(B(x, r))} \int_{B(x, r)}|f| d \mu, \quad x \in X,
$$

and

$$
M f(x)=\sup _{B \ni x} \frac{1}{\mu(B)} \int_{B}|f| d \mu, \quad x \in X,
$$

respectively. Here $B$ refers to any open ball in $(X, \rho)$ and by $B(x, r)$ we denote the open ball centered at $x \in X$ with radius $r>0$.

Recall that an operator $T$ is said to be of strong type $(p, p)$ for some $p \in[1, \infty]$ if $T$ is bounded on $L^{p}=L^{p}(\mathbb{X})$. Similarly, $T$ is of weak type $(p, p)$ if $T$ is bounded from $L^{p}$ to $L^{p, \infty}=L^{p, \infty}(\mathbb{X})$ (we use the convention $\left.L^{\infty, \infty}=L^{\infty}\right)$. Obviously, the operators $M^{c}$ and $M$ are of strong type $(\infty, \infty)$ in case of any metric measure space. Moreover, by using the Marcinkiewicz interpolation theorem, if $M^{c}$ (equivalently $M$ ) is of weak or strong type $\left(p_{0}, p_{0}\right)$ for some $p_{0} \in[1, \infty)$, then it is of strong (and 
hence weak) type $(p, p)$ for every $p>p_{0}$. If the measure is doubling, that is $\mu(B(x, 2 r)) \lesssim \mu(B(x, r))$ uniformly in $x \in X$ and $r>0$, then both $M^{c}$ and $M$ are of weak type $(1,1)$. However, in general, the weak type $(1,1)$ inequalities may not occur. Furthermore, as we will see, it is even possible to construct a space for which the associated operators $M^{c}$ and $M$ are not of weak (and hence strong) type $(p, p)$ for every $p \in[1, \infty)$.

Finding examples of metric measure spaces with specific properties of associated maximal operators is usually a nontrivial task; see Aldaz [1], for example. H.-Q. Li greatly contributed the program of searching spaces which are peculiar from the point of view of mapping properties of maximal operators. In this context, in [2], $[\mathbf{3}]$, and $[\mathbf{4}]$, he considered a class of the cusp spaces. In [2] H.-Q. Li showed that for any fixed $1<p_{0}<\infty$ there exists a space for which the associated operator $M^{c}$ is of strong type $(p, p)$ if and only if $p>p_{0}$. Then, in $[\mathbf{3}]$ examples of spaces were furnished for which $M$ is of strong type $(p, p)$ if and only if $p>p_{0}$. Moreover, for every $1<\tau \leq 2$ there are examples of spaces for which $M^{c}$ is of weak type $(1,1)$, and $M$ is of strong type $(p, p)$ if and only if $p>\tau$. Finally, in [4] H.-Q. Li showed that there are spaces with exponential volume growth for which $M^{c}$ is of weak type $(1,1)$, while $M$ is of strong type $(p, p)$ for every $p>1$.

The aim of this article is to complement and strengthen the results obtained by H.-Q. Li. For a fixed metric measure space $\mathbb{X}$ denote by $P_{s}^{c}$ and $P_{s}$ the sets consisting of such $p \in[1, \infty]$ for which the associated operators, $M^{c}$ and $M$ are of strong type $(p, p)$, respectively. Similarly, let $P_{w}^{c}$ and $P_{w}$ consist of such $p \in[1, \infty]$ for which $M^{c}$ and $M$ are of weak type $(p, p)$, respectively. Then

(i) each of the four sets is of the form $\{\infty\},\left[p_{0}, \infty\right]$, or $\left(p_{0}, \infty\right]$, for some $p_{0} \in[1, \infty)$;

(ii) we have the following inclusions

$$
P_{s} \subset P_{s}^{c}, \quad P_{w} \subset P_{w}^{c}, \quad P_{s}^{c} \subset P_{w}^{c} \subset \overline{P_{s}^{c}}, \quad P_{s} \subset P_{w} \subset \overline{P_{s}},
$$

where $\bar{E}$ denotes the closure of $E$ in the usual topology of $\mathbb{R} \cup\{\infty\}$.

We will show that the conditions above are the only ones that the sets $P_{s}^{c}, P_{s}, P_{w}^{c}$, and $P_{w}$ must satisfy. Namely, we will prove the following:

Theorem 1. Let $P_{s}^{c}, P_{s}, P_{w}^{c}$, and $P_{w}$ be such that the conditions (i) and (ii) hold. Then there exists a (non-doubling) metric measure space for which the associated Hardy-Littlewood maximal operators, centered $M^{c}$ and non-centered $M$, satisfy 
- $M^{c}$ is of strong type $(p, p)$ if and only if $p \in P_{s}^{c}$,

- $M$ is of strong type $(p, p)$ if and only if $p \in P_{s}$,

- $M^{c}$ is of weak type $(p, p)$ if and only if $p \in P_{w}^{c}$,

- $M$ is of weak type $(p, p)$ if and only if $p \in P_{w}$.

The proof of Theorem 1 is postponed to Section 4 .

\section{First generation spaces}

We begin with a construction of some metric measure spaces called by us the first generation spaces. The common property of these spaces is a similarity in the behavior of the associated operators $M^{c}$ and $M$, by what we mean the equalities $P_{s}^{c}=P_{s}$ and $P_{w}^{c}=P_{w}$. We begin with an overview of the first generation spaces and then we consider two subtypes separately in detail.

Let $\tau=\left(\tau_{n}\right)_{n \in \mathbb{N}}$ be a fixed sequence of positive integers. Define

$$
X_{\tau}=\left\{x_{n}: n \in \mathbb{N}\right\} \cup\left\{x_{n i}: i=1, \ldots, \tau_{n}, n \in \mathbb{N}\right\},
$$

where all elements $x_{n}, x_{n i}$ are pairwise different (and located on the plane, say). We define the metric $\rho=\rho_{\tau}$ determining the distance between two different elements $x$ and $y$ by the formula

$$
\rho(x, y)= \begin{cases}1 & \text { if } x_{n} \in\{x, y\} \subset S_{n} \text { for some } n \in \mathbb{N} \\ 2 & \text { in the other case }\end{cases}
$$

By $S_{n}$ we denote the branch $S_{n}=\left\{x_{n}, x_{n 1}, \ldots, x_{n \tau_{n}}\right\}$ and by $S_{n}^{\prime}$ the branch without the root, $S_{n}^{\prime}=S_{n} \backslash\left\{x_{n}\right\}$. Figure 1 shows a model of the space $\left(X_{\tau}, \rho\right)$. The solid line between two points indicates that the distance between them equals 1 . Otherwise the distance equals 2 .

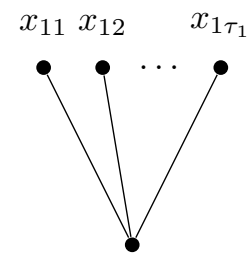

$x_{1}$

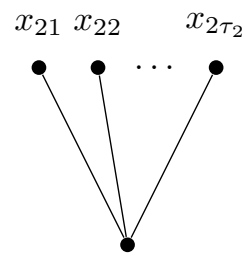

$x_{2}$

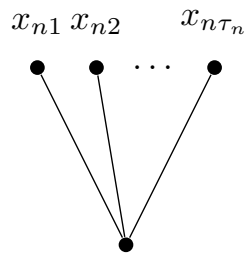

$x_{n}$

Figure 1. 
Note that we can explicitly describe any ball: for $n \in \mathbb{N}$,

$$
B\left(x_{n}, r\right)= \begin{cases}\left\{x_{n}\right\} & \text { for } 0<r \leq 1, \\ S_{n} & \text { for } 1<r \leq 2, \\ X_{\tau} & \text { for } 2<r\end{cases}
$$

and for $i \in\left\{1, \ldots, \tau_{n}\right\}, n \in \mathbb{N}$,

$$
B\left(x_{n i}, r\right)= \begin{cases}\left\{x_{n i}\right\} & \text { for } 0<r \leq 1, \\ \left\{x_{n}, x_{n i}\right\} & \text { for } 1<r \leq 2, \\ X_{\tau} & \text { for } 2<r\end{cases}
$$

We define the measure $\mu=\mu_{\tau, F}$ on $X_{\tau}$ by letting $\mu\left(\left\{x_{n}\right\}\right)=d_{n}$ and $\mu\left(\left\{x_{n i}\right\}\right)=d_{n} F(n, i)$, where $F>0$ is a given function and $d=\left(d_{n}\right)_{n \in \mathbb{N}}$ is an appropriate sequence of strictly positive numbers with $d_{1}=1$ and $d_{n}$ chosen (uniquely!) in such a way that $\mu\left(S_{n}\right)=\mu\left(S_{n-1}\right) / 2, n \geq 2$. Note that this implies $\mu\left(X_{\tau}\right)<\infty$. Moreover, observe that $\mu$ is non-doubling. From now on we shall use the sign $|E|$ instead of $\mu(E)$ for $E \subset X_{\tau}$. It will be clear from the context when the symbol $|\cdot|$ refers to the measure and when it denotes the absolute value sign.

For a function $f$ on $X_{\tau}$ (which is in fact a 'sequence' of numbers) the Hardy-Littlewood maximal operators, centered $M^{c}$ and non-centered $M$, are given by

$$
M^{c} f(x)=\sup _{r>0} \frac{1}{|B(x, r)|} \sum_{y \in B(x, r)}|f(y)| \cdot|\{y\}|, \quad x \in X_{\tau},
$$

and

$$
M f(x)=\sup _{B \ni x} \frac{1}{|B|} \sum_{y \in B}|f(y)| \cdot|\{y\}|, \quad x \in X_{\tau},
$$

respectively. In this setting $M$ is of weak type $(p, p)$ for some $1 \leq$ $p<\infty$ if $\|M f\|_{p, \infty} \lesssim\|f\|_{p}$ uniformly in $f \in \ell^{p}\left(X_{\tau}, \mu\right)$, where $\|g\|_{p}=$ $\left(\sum_{x \in X_{\tau}}|g(x)|^{p}|\{x\}|\right)^{1 / p}$ and $\|g\|_{p, \infty}=\sup _{\lambda>0} \lambda\left|E_{\lambda}(g)\right|^{1 / p}$ with $E_{\lambda}(g)=$ $\left\{x \in X_{\tau}:|g(x)|>\lambda\right\}$. Similarly, $M$ is of strong type $(p, p)$ for some $1 \leq p \leq \infty$ if $\|M f\|_{p} \lesssim\|f\|_{p}$ uniformly in $f \in \ell^{p}\left(X_{\tau}, \mu\right)$, where $\|g\|_{\infty}=$ $\sup _{x \in X_{\tau}}|g(x)|$. Here the notation $A \lesssim B$ is used to indicate that $A \leq$ $C B$ with a positive constant $C$ independent of significant quantities. Moreover, for given a function $f \geq 0$ and a set $E \subset X_{\tau}$ we denote the average value of $f$ on $E$ by

$$
A_{E}(f)=\frac{1}{|E|} \sum_{x \in E} f(x)|\{x\}| .
$$


Analogous definitions and comments apply to $M^{c}$ instead of $M$ and then to both $M$ and $M^{c}$ in the context of the space $\left(Y_{\tau}, \mu\right)$ in Section 3.

We are ready to describe two subtypes of the first generation spaces.

2.1. We first construct and investigate first generation spaces for which the equalities $P_{s}^{c}=P_{s}$ and $P_{w}^{c}=P_{w}$ hold and, in addition, there is no significant difference between the incidence of the weak and strong type inequalities, by what we mean that $P_{s}^{c}=P_{w}^{c}$ and $P_{s}=P_{w}$. Of course, combining all these equalities, we obtain that for such spaces all four sets take the same form. In the first step, for any fixed $p_{0} \in[1, \infty]$ we construct a space denoted by $\hat{\mathbb{X}}_{p_{0}}$ for which $P_{s}^{c}=P_{s}=P_{w}^{c}=P_{w}=$ $\left[p_{0}, \infty\right]$ (by $[\infty, \infty]$ we mean $\left.\{\infty\}\right)$. Then, after slight modifications, for any fixed $p_{0} \in[1, \infty)$ we get a space $\hat{\mathbb{X}}_{p_{0}}^{\prime}$ for which $P_{s}^{c}=P_{s}=P_{w}^{c}=$ $P_{w}=\left(p_{0}, \infty\right]$.

Fix $p_{0} \in[1, \infty]$ and let $\hat{\mathbb{X}}_{p_{0}}=\left(X_{\tau}, \rho, \mu\right)$ be the first generation space with $\tau_{n}=\left\lfloor\frac{(n+1)^{p_{0}}}{n}\right\rfloor$ in the case $p_{0} \in[1, \infty)$, or $\tau_{n}=2^{n}$ in the case $p_{0}=\infty$, and $F(n, i)=n, i=1, \ldots, \tau_{n}, n \in \mathbb{N}$. The key point for considerations that follow is that we have: for $p_{0} \neq 1$,

$$
\lim _{n \rightarrow \infty} \frac{n \tau_{n}}{(n+1)^{p}}=\infty, \quad 1 \leq p<p_{0},
$$

and for $p_{0} \neq \infty$,

$$
\frac{n \tau_{n}}{(n+1)^{p_{0}}} \leq 1, \quad n \in \mathbb{N}
$$

Proposition 2. Fix $p_{0} \in[1, \infty]$ and let $\hat{\mathbb{X}}_{p_{0}}$ be the metric measure space defined above. Then the associated maximal operators, centered $M^{c}$ and non-centered $M$, are not of weak type $(p, p)$ for $1 \leq p<p_{0}$, but are of strong type $(p, p)$ for $p \geq p_{0}$.

Proof: It suffices to prove that $M^{c}$ fails to be of weak type $(p, p)$ for $1 \leq p<p_{0}$ and $M$ is of strong type $\left(p_{0}, p_{0}\right)$. First we show that $M^{c}$ is not of weak type $(p, p)$ for $1 \leq p<p_{0}$. Consider $p_{0} \in(1, \infty]$ and fix $p \in$ $\left[1, p_{0}\right)$. Let $f_{n}=\delta_{x_{n}}, n \geq 1$. Then $\left\|f_{n}\right\|_{p}^{p}=d_{n}$ and $M^{c} f_{n}\left(x_{n i}\right) \geq \frac{1}{n+1}$, $i=1, \ldots, \tau_{n}$. This implies that $\left|E_{1 /(2(n+1))}\left(M^{c} f_{n}\right)\right| \geq n \tau_{n} d_{n}$ and hence

$$
\limsup _{n \rightarrow \infty} \frac{\left\|M^{c} f_{n}\right\|_{p, \infty}^{p}}{\left\|f_{n}\right\|_{p}^{p}} \geq \lim _{n \rightarrow \infty} \frac{n \tau_{n} d_{n}}{(2(n+1))^{p} d_{n}}=\infty .
$$

In the next step we show that $M$ is of strong type $\left(p_{0}, p_{0}\right)$. Consider $p_{0} \in[1, \infty)$, since the case $p_{0}=\infty$ is trivial. Let $f \in \ell^{p_{0}}\left(\hat{\mathbb{X}}_{p_{0}}\right)$. Without any loss of generality we assume that $f \geq 0$. Denote $\mathcal{D}=\left\{\left\{x_{n}, x_{n i}\right\}\right.$ : 
$\left.n \in \mathbb{N}, i=1, \ldots, \tau_{n}\right\}$. We use the estimate

$$
\|M f\|_{p_{0}}^{p_{0}} \leq \sum_{B \subset X_{\tau}} \sum_{x \in B} A_{B}(f)^{p_{0}}|\{x\}|=\sum_{B \subset X_{\tau}} A_{B}(f)^{p_{0}}|B| .
$$

Note that each $x \in X_{\tau}$ belongs to at most three different balls which are not elements of $\mathcal{D}$. Combining this with Hölder's inequality, we obtain

$$
\sum_{B \notin \mathcal{D}} A_{B}(f)^{p_{0}}|B| \leq \sum_{B \notin \mathcal{D}} \sum_{x \in B} f(x)^{p_{0}}|\{x\}| \leq 3\|f\|_{p_{0}}^{p_{0}} .
$$

Therefore

$$
\|M f\|_{p_{0}}^{p_{0}} \leq 3\|f\|_{p_{0}}^{p_{0}}+\sum_{n \in \mathbb{N}} \sum_{i=1}^{\tau_{n}}\left(\frac{f\left(x_{n}\right)+n f\left(x_{n i}\right)}{n+1}\right)^{p_{0}}\left|\left\{x_{n}, x_{n i}\right\}\right| .
$$

Finally, we use the inequalities $\left(f\left(x_{n}\right)+n f\left(x_{n i}\right)\right)^{p_{0}} \leq\left(2 f\left(x_{n}\right)\right)^{p_{0}}+$ $\left(2 n f\left(x_{n i}\right)\right)^{p_{0}}$ and $\left|\left\{x_{n}, x_{n i}\right\}\right| \leq 2\left|\left\{x_{n i}\right\}\right|=2 n\left|\left\{x_{n}\right\}\right|$ to estimate the double sum in (1) by

$$
\begin{array}{r}
2^{p_{0}+1}\left(\sum_{n \in \mathbb{N}} \frac{n \tau_{n}}{(n+1)^{p_{0}}} f\left(x_{n}\right)^{p_{0}}\left|\left\{x_{n}\right\}\right|+\sum_{n \in \mathbb{N}} \sum_{i=1}^{\tau_{n}}\left(\frac{n f\left(x_{n i}\right)}{n+1}\right)^{p_{0}}\left|\left\{x_{n i}\right\}\right|\right) \\
\leq 2^{p_{0}+1}\|f\|_{p_{0}}^{p_{0}} .
\end{array}
$$

A modification of arguments from the proof of Proposition 2 shows that, for a fixed $p_{0} \in[1, \infty)$, replacing the former $\tau_{n}$ by $\tau_{n}^{\prime}=$ $\left\lfloor(\log (n)+1) \frac{(n+1)^{p_{0}}}{n}\right\rfloor$ leads to the space $\hat{\mathbb{X}}_{p_{0}}^{\prime}$ for which $P_{s}^{c}=P_{s}=$ $P_{w}^{c}=P_{w}=\left(p_{0}, \infty\right]$. Moreover, it may be noted that only the properties $\lim _{n \rightarrow \infty} \frac{n \tau_{n}^{\prime}}{(n+1)^{p}}=\infty, 1 \leq p \leq p_{0}$, and $\sup _{n \in \mathbb{N}} \frac{n \tau_{n}^{\prime}}{(n+1)^{p}}<\infty, p>p_{0}$, are essential.

2.2. In contrast to the former case, for the spaces we now construct and study, the equalities $P_{s}^{c}=P_{s}$ and $P_{w}^{c}=P_{w}$ still hold, but there is a difference between the incidence of the weak and strong type inequalities. For any fixed $p_{0} \in[1, \infty)$ we construct a space denoted by $\widetilde{\mathbb{X}}_{p_{0}}$ for which $P_{s}^{c}=P_{s}=\left(p_{0}, \infty\right]$ and $P_{w}^{c}=P_{w}=\left[p_{0}, \infty\right]$. We begin with the case $p_{0}=1$, which is discussed separately because it is relatively simple and may be helpful to outline the core idea behind the more difficult case $p_{0} \in(1, \infty)$.

By $\widetilde{\mathbb{X}}_{1}$ we denote the first generation space $\left(X_{\tau}, \rho, \mu\right)$ with construction based on $\tau_{n}=n$ and $F(n, i)=2^{i}$. Recall that $\mu$ is non-doubling. 
Proposition 3. Let $\widetilde{\mathbb{X}}_{1}$ be the metric measure space defined above. Then the associated maximal operators, centered $M^{c}$ and non-centered $M$, are not of strong type $(1,1)$, but are of weak type $(1,1)$.

Proof: First we note that $M^{c}$ fails to be of strong type $(1,1)$. Indeed, let $f_{n}=\delta_{x_{n}}, n \geq 1$. Then $\left\|f_{n}\right\|_{1}=d_{n}$ and for $i=1, \ldots, n$ we have $M^{c} f_{n}\left(x_{n i}\right) \geq\left(1+2^{i}\right)^{-1}>1 / 2^{i+1}$ and hence $\left\|M^{c} f_{n}\right\|_{1} \geq$ $\sum_{i=1}^{n} 2^{i} d_{n} / 2^{i+1}=n\left\|f_{n}\right\|_{1} / 2$.

In the next step we show that $M$ is of weak type $(1,1)$. Let $f \in \ell^{1}\left(\widetilde{\mathbb{X}}_{1}\right)$, $f \geq 0$, and consider $\lambda>0$ such that $E_{\lambda}(M f) \neq \emptyset$. If $\lambda<A_{X_{\tau}}(f)$, then $\lambda\left|E_{\lambda}(M f)\right| /\|f\|_{1} \leq 1$ follows. Therefore, from now on assume that $\lambda \geq A_{X_{\tau}}(f)$. With this assumption, if for some $x \in S_{n}$ we have $M f(x)>\lambda$, then any ball $B$ containing $x$ and realizing $A_{B}(f)>\lambda$ must be a subset of $S_{n}$. Take any $n \in \mathbb{N}$ such that $E_{\lambda}(M f) \cap S_{n} \neq \emptyset$. If $\lambda<A_{S_{n}}(f)$, then

$$
\frac{\lambda\left|E_{\lambda}(M f) \cap S_{n}\right|}{\sum_{x \in S_{n}} f(x)|\{x\}|} \leq 1 .
$$

Assume that $\lambda \geq A_{S_{n}}(f)$ and take $x \in E_{\lambda}(M f) \cap S_{n}$. Now, any ball $B$ containing $x$ and realizing $A_{B}(f)>\lambda$ must be a proper subset of $S_{n}$. If $E_{\lambda}(M f) \cap S_{n}^{\prime}=\emptyset$, then $x=x_{n}$ so we obtain $f\left(x_{n}\right)>\lambda$ and hence (2) again follows. In the opposite case, if $E_{\lambda}(M f) \cap S_{n}^{\prime} \neq \emptyset$, denote $j=\max \left\{i \in\{1, \ldots, n\}: M f\left(x_{n i}\right)>\lambda\right\}$. Then $f\left(x_{n j}\right)>\lambda$ or $\frac{f\left(x_{n}\right)\left|\left\{x_{n}\right\}\right|+f\left(x_{n j}\right)\left|\left\{x_{n j}\right\}\right|}{\left|\left\{x_{n}\right\}\right|+\left|\left\{x_{n j}\right\}\right|}>\lambda$. Therefore, $f\left(x_{n}\right)\left|\left\{x_{n}\right\}\right|+f\left(x_{n j}\right)\left|\left\{x_{n j}\right\}\right|>$ $\lambda\left|\left\{x_{n j}\right\}\right|$ and combining this with the estimate $\left|E_{\lambda}(M f) \cap S_{n}\right| \leq 2\left|\left\{x_{n j}\right\}\right|$, we obtain

$$
\frac{\lambda\left|E_{\lambda}(M f) \cap S_{n}\right|}{\sum_{x \in S_{n}} f(x)|\{x\}|} \leq \frac{2 \lambda\left|\left\{x_{n j}\right\}\right|}{f\left(x_{n}\right)\left|\left\{x_{n}\right\}\right|+f\left(x_{n j}\right) \mid\left\{x_{n j} \mid\right\}} \leq 2 .
$$

Since $\frac{\lambda\left|E_{\lambda}(M f) \cap S_{n}\right|}{\sum_{x \in S_{n}} f(x)|\{x\}|} \leq 2$ for any branch $S_{n}$ such that $E_{\lambda}(M f) \cap S_{n} \neq$ $\emptyset$, we have

$$
\frac{\lambda\left|E_{\lambda}(M f)\right|}{\|f\|_{1}} \leq 2
$$

and, consequently, the weak type $(1,1)$ estimate $\|M f\|_{1, \infty} \leq 2\|f\|_{1}$ follows.

Now fix $p_{0} \in(1, \infty)$ and consider $\widetilde{\mathbb{X}}_{p_{0}}=\left(X_{\tau}, \rho, \mu\right)$, with construction based on $\tau_{n}=\tau_{n}\left(p_{0}\right)$ and $F(n, i)=F_{p_{0}}(n, i)$, defined as follows. Let $c_{n}=\left\lfloor\frac{(n+1)^{p_{0}}}{n}\right\rfloor$ and

$$
e_{n}=\max \left\{k \in \mathbb{N}: 2^{k-1} \leq c_{n} \text { and } 2^{1-k-p_{0}} \geq\left(\frac{1}{1+n}\right)^{p_{0}}\right\}, \quad n \in \mathbb{N} .
$$


Note that $\lim _{n \rightarrow \infty} e_{n}=\infty$. Then, for $j \in\left\{1, \ldots, e_{n}\right\}, n \in \mathbb{N}$, define $m_{n j}$ by the equality

$$
2^{1-j}\left(\frac{1}{1+m_{n j}}\right)^{p_{0}}=\left(\frac{1}{1+n}\right)^{p_{0}}
$$

and $s_{n j}$ by

$$
s_{n j}=\min \left\{k \in \mathbb{N}: k m_{n j} \geq 2^{1-j} n c_{n}\right\} .
$$

Observe that for $j \in\left\{1, \ldots, e_{n}\right\}, n \in \mathbb{N}$,

$$
1 \leq m_{n j} \leq n, \quad 2^{1-j} n c_{n} \leq s_{n j} m_{n j} \leq 2^{2-j} n c_{n} .
$$

Finally, denote $\tau_{n}=\sum_{j=1}^{e_{n}} s_{n j}, n \in \mathbb{N}$, and $F(n, i)=m_{n j(n, i)}, i=$ $1, \ldots, \tau_{n}, n \in \mathbb{N}$, where

$$
j(n, i)=\min \left\{k \in\left\{1, \ldots, e_{n}\right\}: \sum_{j=1}^{k} s_{n j} \geq i\right\} .
$$

Proposition 4. Let $\widetilde{\mathbb{X}}_{p_{0}}$ be the metric measure space defined above. Then the associated maximal operators, centered $M^{c}$ and non-centered $M$, are not of strong type $\left(p_{0}, p_{0}\right)$, but are of weak type $\left(p_{0}, p_{0}\right)$.

Proof: First we note that $M^{c}$ is not of strong type $\left(p_{0}, p_{0}\right)$. Indeed, let $f_{n}=\delta_{x_{n}}, n \geq 1$. Then $\left\|f_{n}\right\|_{p_{0}}^{p_{0}}=d_{n}$ and for $i=1, \ldots, \tau_{n}$ we have $M^{c} f_{n}\left(x_{n i}\right) \geq\left(1+m_{n j(n, i)}\right)^{-1}$ and hence

$$
\begin{aligned}
\left\|M^{c} f_{n}\right\|_{p_{0}}^{p_{0}} & \geq \sum_{j=1}^{e_{n}} \sum_{k=1}^{s_{n j}}\left(\frac{1}{1+m_{n j}}\right)^{p_{0}} d_{n} m_{n j}=d_{n} \sum_{j=1}^{e_{n}} \frac{s_{n j} m_{n j}}{\left(1+m_{n j}\right)^{p_{0}}} \\
& \geq d_{n} \sum_{j=1}^{e_{n}} \frac{2^{1-j} n c_{n}}{\left(1+m_{n j}\right)^{p_{0}}}=d_{n} \sum_{j=1}^{e_{n}} \frac{n c_{n}}{(1+n)^{p_{0}}}=e_{n} \frac{n c_{n}}{(1+n)^{p_{0}}}\left\|f_{n}\right\|_{p_{0}}^{p_{0}} .
\end{aligned}
$$

Since $\lim _{n \rightarrow \infty} e_{n}=\infty$ and $\lim _{n \rightarrow \infty} \frac{n c_{n}}{(1+n)^{p_{0}}}=1$, we are done.

In the next step we show that $M$ is of weak type $\left(p_{0}, p_{0}\right)$. Let $f \in$ $\ell^{p_{0}}\left(\widetilde{\mathbb{X}}_{p_{0}}\right), f \geq 0$, and consider $\lambda>0$ such that $E_{\lambda}(M f) \neq \emptyset$. If $\lambda<$ $A_{X_{\tau}}(f)$, then using the inequality $\|f\|_{1} \leq\|f\|_{p_{0}}\left|X_{\tau}\right|^{1 / q_{0}}$, where $q_{0}$ is the exponent conjugate to $p_{0}$, we obtain $\lambda^{p_{0}}\left|E_{\lambda}(M f)\right| /\|f\|_{p_{0}}^{p_{0}}<1$. Therefore, from now on assume that $\lambda \geq A_{X_{\tau}}(f)$. Take any $n \in \mathbb{N}$ such that $E_{\lambda}(M f) \cap S_{n} \neq \emptyset$. If $\lambda<A_{S_{n}}(f)$, then using similar argument as above we have

$$
\frac{\lambda^{p_{0}}\left|E_{\lambda}(M f) \cap S_{n}\right|}{\sum_{x \in S_{n}} f(x)^{p_{0}}|\{x\}|} \leq 1
$$


Assume that $\lambda \geq A_{S_{n}}(f)$. If $E_{\lambda}(M f) \cap S_{n}^{\prime}=\emptyset$, then $f\left(x_{n}\right)>\lambda$ and hence (3) again follows. In the opposite case, we have $\left|E_{\lambda}(M f) \cap S_{n}\right| \leq$ $2\left|E_{\lambda}(M f) \cap S_{n}^{\prime}\right|$. Assume that $f\left(x_{n}\right)<\left(1+m_{n e_{n}}\right) \lambda / 2$. If $x \in E_{\lambda}(M f) \cap$ $S_{n}^{\prime}$, then $f(x) \geq \lambda / 2$ and hence

$$
\frac{\lambda^{p_{0}}\left|E_{\lambda}(M f) \cap S_{n}\right|}{\sum_{x \in S_{n}} f(x)^{p_{0}}|\{x\}|} \leq \frac{2 \lambda^{p_{0}}\left|E_{\lambda}(M f) \cap S_{n}^{\prime}\right|}{\sum_{x \in S_{n}} f(x)^{p_{0}}|\{x\}|} \leq 2^{p_{0}+1} .
$$

Otherwise, if $f\left(x_{n}\right) \geq\left(1+m_{n e_{n}}\right) \lambda / 2$, denote $r=\min \left\{j \in\left\{1, \ldots, e_{n}\right\}\right.$ : $\left.f\left(x_{n}\right) \geq\left(1+m_{n j}\right) \lambda / 2\right\}$. Let $S_{n}^{(r)}=\left\{x_{n i}: i \in\left\{1, \ldots, \sum_{j=1}^{r-1} s_{n j}\right\}\right\}$. Note that the case $S_{n}^{(r)}=\emptyset$ is possible. Assume that $S_{n}^{(r)} \neq \emptyset$. If $x \in E_{\lambda}(M f) \cap S_{n}^{(r)}$, then $f(x)>\lambda / 2$ and hence

$$
\frac{\lambda^{p_{0}}\left|E_{\lambda}(M f) \cap S_{n}^{(r)}\right|}{\sum_{x \in S_{n}^{(r)}} f(x)^{p_{0}}|\{x\}|} \leq 2^{p_{0}+1}
$$

Moreover, we have

$$
\begin{aligned}
\frac{\lambda^{p_{0}}\left|E_{\lambda}(M f) \cap\left(S_{n} \backslash S_{n}^{(r)}\right)\right|}{f\left(x_{n}\right)^{p_{0}}\left|\left\{x_{n}\right\}\right|} & \leq\left(\frac{2}{1+m_{n r}}\right)^{p_{0}} \frac{\left|S_{n} \backslash S_{n}^{(r)}\right|}{\left|\left\{x_{n}\right\}\right|} \\
& \leq\left(\frac{2}{1+m_{n r}}\right)^{p_{0}} \frac{2\left|\left(S_{n} \backslash S_{n}^{(r)}\right) \cap S_{n}^{\prime}\right|}{\left|\left\{x_{n}\right\}\right|} \\
& \leq\left(\frac{2}{1+m_{n r}}\right)^{p_{0}} 2 \sum_{j=r}^{e_{n}} n c_{n} 2^{2-j} \\
& <2^{p_{0}+4-r} n c_{n}\left(\frac{1}{1+m_{n r}}\right)^{p_{0}} \\
& =2^{p_{0}+3} \frac{n c_{n}}{(1+n)^{p_{0}}} \leq 2^{p_{0}+3}
\end{aligned}
$$

Therefore, regardless of the posibilities, $S_{n}^{(r)}=\emptyset$ or $S_{n}^{(r)} \neq \emptyset$, we obtain $\frac{\lambda^{p_{0}}\left|E_{\lambda}(M f) \cap S_{n}\right|}{\sum_{x \in S_{n}} f(x)^{p_{0}}|\{x\}|} \leq 2^{p_{0}+3}$. Since $\lambda^{p_{0}}\left|E_{\lambda}(M f) \cap S_{n}\right| / \sum_{x \in S_{n}} f(x)^{p_{0}}|\{x\}| \leq$ $2^{p_{0}+3}$ for any branch $S_{n}$ such that $E_{\lambda}(M f) \cap S_{n} \neq \emptyset$, we have $\lambda^{p_{0}}\left|E_{\lambda}(M f)\right| /\|f\|_{p_{0}}^{p_{0}} \leq 2^{p_{0}+3}$ and, consequently, $\|M f\|_{p_{0}, \infty}^{p_{0}} \leq 2^{p_{0}+3}\|f\|_{p_{0}}^{p_{0}}$.

\section{Second generation spaces}

Now we construct and study metric measure spaces called by us the second generation spaces. The common attribute of these spaces is a significant difference in the behavior of the associated operators $M^{c}$ and $M$, 
by what we mean that $M^{c}$ is of strong type $(1,1)$, which implies the basic property $P_{s}^{c}=P_{w}^{c}=[1, \infty]$, while $P_{s}$ (and possibly $P_{w}$ ) is a proper subset of $[1, \infty]$. Let $\tau=\left(\tau_{n}\right)_{n \in \mathbb{N}}$ be a fixed sequence of positive integers. Define

$$
Y_{\tau}=\left\{y_{n}: n \in \mathbb{N}\right\} \cup\left\{y_{n i}, y_{n i}^{\prime}: i=1, \ldots, \tau_{n}, n \in \mathbb{N}\right\},
$$

where all elements $y_{n}, y_{n i}, y_{n i}^{\prime}$ are pairwise different. We define the metric $\rho=\rho_{\tau}$ determining the distance between two different elements $x$ and $y$ by the formula

$$
\rho(x, y)= \begin{cases}1 \quad & \text { if }\{x, y\}=T_{n i} \text { or } y_{n} \in\{x, y\} \subset T_{n} \backslash T_{n}^{\prime} \\ & \text { for some } n \in \mathbb{N}, i \in\left\{1, \ldots, \tau_{n}\right\} \\ 2 \quad \text { in the other case. }\end{cases}
$$

By $T_{n}$ we denote the branch $T_{n}=\left\{y_{n}, y_{n 1}, \ldots, y_{n \tau_{n}}, y_{n 1}^{\prime}, \ldots, y_{n \tau_{n}}^{\prime}\right\}$. Additionally, we denote $T_{n}^{\prime}=\left\{y_{n 1}^{\prime}, \ldots, y_{n \tau_{n}}^{\prime}\right\}$ and $T_{n i}=\left\{y_{n i}, y_{n i}^{\prime}\right\}$. Figure 2 shows a model of the space $\left(Y_{\tau}, \rho\right)$.

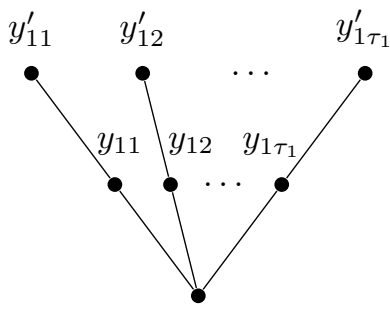

$y_{1}$

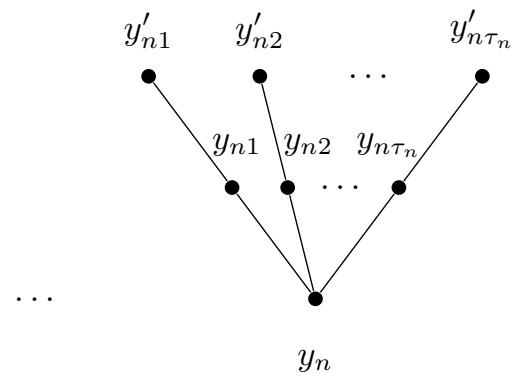

FiguRE 2.

Note that we can explicitly describe any ball: for $n \in \mathbb{N}$,

$$
B\left(y_{n}, r\right)= \begin{cases}\left\{y_{n}\right\} & \text { for } 0<r \leq 1 \\ T_{n} \backslash T_{n}^{\prime} & \text { for } 1<r \leq 2 \\ Y_{\tau} & \text { for } 2<r\end{cases}
$$

and for $i \in\left\{1, \ldots, \tau_{n}\right\}, n \in \mathbb{N}$,

$$
B\left(y_{n i}, r\right)= \begin{cases}\left\{y_{n i}\right\} & \text { for } 0<r \leq 1 \\ \left\{y_{n}\right\} \cup T_{n i} & \text { for } 1<r \leq 2 \\ Y_{\tau} & \text { for } 2<r\end{cases}
$$


and

$$
B\left(y_{n i}^{\prime}, r\right)= \begin{cases}\left\{y_{n i}^{\prime}\right\} & \text { for } 0<r \leq 1 \\ T_{n i} & \text { for } 1<r \leq 2 \\ Y_{\tau} & \text { for } 2<r\end{cases}
$$

We define the measure $\mu=\mu_{\tau, F}$ by letting $\mu\left(\left\{y_{n}\right\}\right)=d_{n}, \mu\left(\left\{y_{n i}\right\}\right)=$ $\frac{d_{n}}{\tau_{n}}$, and $\mu\left(\left\{y_{n i}^{\prime}\right\}\right)=d_{n} F(n, i)$, where $F>0$ is a given function and $d=\left(d_{n}\right)_{n \in \mathbb{N}}$ is an appropriate sequence of strictly positive numbers with $d_{1}=1$ and $d_{n}$ chosen (uniquely!) in such a way that $\left|T_{n}\right|=$ $\left|T_{n-1}\right| / 2, n \geq 2$. Note that this implies $\left|Y_{\tau}\right|<\infty$ and observe that $\mu$ is non-doubling.

We are ready to describe two subtypes of the second generation spaces.

3.1. We first construct spaces for which apart from the basic property $P_{s}^{c}=P_{w}^{c}=[1, \infty]$ we also have $P_{s}=P_{w}$. In the first step, for any fixed $p_{0} \in(1, \infty]$ we construct a space denoted by $\hat{\mathbb{Y}}_{p_{0}}$ for which $P_{s}=P_{w}=$ $\left[p_{0}, \infty\right]$. Then, after a slight modification, for any fixed $p_{0} \in[1, \infty)$ we get a space $\hat{\mathbb{Y}}_{p_{0}}^{\prime}$ for which $P_{s}=P_{w}=\left(p_{0}, \infty\right]$.

Fix $p_{0} \in(1, \infty]$ and let $\hat{\mathbb{Y}}_{p_{0}}$ be the second generation space with $\tau_{n}=\left\lfloor\frac{(n+1)^{p_{0}}}{n}\right\rfloor$ in the case $p_{0} \in(1, \infty)$, or $\tau_{n}=2^{n}$ when $p_{0}=\infty$, and $F(n, i)=n, i=1, \ldots, \tau_{n}, n \in \mathbb{N}$.

Proposition 5. Let $\hat{\mathbb{Y}}_{p_{0}}$ be the metric measure space defined above. Then the associated centered maximal operator $M^{c}$ is of strong type $(1,1)$, while the non-centered $M$ is not of weak type $(p, p)$ for $1 \leq p<p_{0}$, but is of strong type $(p, p)$ for $p \geq p_{0}$.

Proof: First we show that $M^{c}$ is of strong type $(1,1)$. Let $f \in \ell^{1}\left(\hat{\mathbb{Y}}_{p_{0}}\right)$, $f \geq 0$. Denote $\mathcal{G}=\left\{\left\{y_{n}\right\} \cup T_{n i}: n \in \mathbb{N}, i=1, \ldots, \tau_{n}\right\}$ and $\mathcal{B}_{y}=$ $\left\{B\left(y, \frac{1}{2}\right), B\left(y, \frac{3}{2}\right), B\left(y, \frac{5}{2}\right)\right\}, y \in Y_{\tau}$. We use the estimate

$$
\left\|M^{c} f\right\|_{1} \leq \sum_{y \in Y_{\tau}} \sum_{B \in \mathcal{B}_{y}} A_{B}(f)|\{y\}| .
$$

Note that each $y \in Y_{\tau}$ belongs to at most four different balls which are not elements of $\mathcal{G}$. Thus we obtain

$$
\sum_{y \in Y_{\tau}} \sum_{B \in \mathcal{B}_{y} \backslash \mathcal{G}} A_{B}(f)|\{y\}| \leq \sum_{B \notin \mathcal{G}} \sum_{y \in B} f(y)|\{y\}| \leq 4\|f\|_{1} .
$$

Therefore

$$
\left\|M^{c} f\right\|_{1} \leq 4\|f\|_{1}+\sum_{n \in \mathbb{N}} \sum_{i=1}^{\tau_{n}} A_{B\left(y_{n i}, \frac{3}{2}\right)}(f)\left|\left\{y_{n i}\right\}\right| .
$$


It suffices to see that the last term of the above expression is estimated by

$$
\sum_{n \in \mathbb{N}} \tau_{n} f\left(y_{n}\right)\left|\left\{y_{n 1}\right\}\right|+\sum_{n \in \mathbb{N}} \sum_{i=1}^{\tau_{n}}\left(f\left(y_{n i}\right)\left|\left\{y_{n i}\right\}\right|+f\left(y_{n i}^{\prime}\right)\left|\left\{y_{n i}^{\prime}\right\}\right|\right)=\|f\|_{1}
$$

In the next step we show that $M$ is not of weak type $(p, p)$ for $1 \leq$ $p<p_{0}$. Indeed, fix $p<p_{0}$ and let $f_{n}=\delta_{y_{n}}, n \geq 1$. Then $\left\|f_{n}\right\|_{p}^{p}=d_{n}$ and $M f_{n}\left(y_{n i}^{\prime}\right) \geq \frac{1}{n+1+\left(1 / \tau_{n}\right)} \geq \frac{1}{n+2}, i=1, \ldots, \tau_{n}$. This implies that $\left|E_{1 /(2(n+2))}\left(M f_{n}\right)\right| \geq n \tau_{n} d_{n}$ and hence we obtain

$$
\limsup _{n \rightarrow \infty} \frac{\left\|M f_{n}\right\|_{p, \infty}^{p}}{\left\|f_{n}\right\|_{p}^{p}} \geq \lim _{n \rightarrow \infty} \frac{n \tau_{n} d_{n}}{(2(n+2))^{p} d_{n}}=\infty
$$

To complete the proof, it suffices to show that $M$ is of strong type $\left(p_{0}, p_{0}\right)$ in the case $p_{0} \in(1, \infty)$. Let $f \in \ell^{p_{0}}\left(\hat{\mathbb{Y}}_{p_{0}}\right), f \geq 0$. We use the estimate

$$
\|M f\|_{p_{0}}^{p_{0}} \leq \sum_{B \subset Y_{\tau}} \sum_{y \in B} A_{B}(f)^{p_{0}}|\{y\}|=\sum_{B \subset Y_{\tau}} A_{B}(f)^{p_{0}}|B| .
$$

Once again note that each $y \in Y_{\tau}$ belongs to at most four different balls which are not elements of $\mathcal{G}$. Combining this with Hölder's inequality, we obtain

$$
\sum_{B \notin \mathcal{G}} A_{B}(f)^{p_{0}}|B| \leq \sum_{B \notin \mathcal{G}} \sum_{y \in B} f(y)^{p_{0}}|\{y\}| \leq 4\|f\|_{p_{0}}^{p_{0}}
$$

Therefore

$$
\begin{aligned}
& \|M f\|_{p_{0}}^{p_{0}} \leq 4\|f\|_{p_{0}}^{p_{0}} \\
& \quad+\sum_{n \in \mathbb{N}} \sum_{i=1}^{\tau_{n}}\left(\frac{f\left(y_{n}\right)+1 / \tau_{n} f\left(y_{n i}\right)+n f\left(y_{n i}^{\prime}\right)}{1+1 / \tau_{n}+n}\right)^{p_{0}}\left|\left\{y_{n}, y_{n i}, y_{n i}^{\prime}\right\}\right| .
\end{aligned}
$$

Finally, we use the inequalities

$$
\begin{aligned}
\left(f\left(y_{n}\right)+1 / \tau_{n} f\left(y_{n i}\right)\right. & \left.+n f\left(y_{n i}^{\prime}\right)\right)^{p_{0}} \\
\leq & \left(3 f\left(y_{n}\right)\right)^{p_{0}}+\left(3 f\left(y_{n i}\right) / \tau_{n}\right)^{p_{0}}+\left(3 n f\left(y_{n i}^{\prime}\right)\right)^{p_{0}}
\end{aligned}
$$


and $\left|\left\{y_{n}, y_{n i}, y_{n i}^{\prime}\right\}\right| \leq 3\left|\left\{y_{n i}^{\prime}\right\}\right|=3 n\left|\left\{y_{n}\right\}\right|$ to estimate the double sum in (4) by

$$
\begin{aligned}
& 3^{p_{0}+1}\left(\sum_{n \in \mathbb{N}} \frac{n \tau_{n} f\left(y_{n}\right)^{p_{0}}}{(n+1)^{p_{0}}}\left|\left\{y_{n}\right\}\right|\right. \\
& \left.\quad+\sum_{n \in \mathbb{N}} \sum_{i=1}^{\tau_{n}} \frac{\left(f\left(y_{n i}\right) / \tau_{n}\right)^{p_{0}}+\left(n f\left(y_{n i}^{\prime}\right)\right)^{p_{0}}}{\left(1+1 / \tau_{n}+n\right)^{p_{0}}}\left|\left\{y_{n i}^{\prime}\right\}\right|\right) \leq 3^{p_{0}+1}\|f\|_{p_{0}}^{p_{0}} .
\end{aligned}
$$

Note that in the same way as it was done at the end of Subsection 2.1, replacing the former $\tau_{n}$ by $\tau_{n}^{\prime}=\left\lfloor(\log (n)+1) \frac{(n+1)^{p_{0}}}{n}\right\rfloor, p_{0} \in[1, \infty)$, results in obtaining the space $\hat{\mathbb{Y}}_{p_{0}}^{\prime}$ for which $P_{s}=P_{w}=\left(p_{0}, \infty\right]$.

3.2. In contrast to the former case the spaces we now construct, apart from the basic property $P_{s}^{c}=P_{w}^{c}=[1, \infty]$, satisfy $P_{s} \varsubsetneqq P_{w}$. Namely, for any fixed $p_{0} \in[1, \infty)$ we construct a space $\widetilde{\mathbb{Y}}_{p_{0}}$ for which $P_{s}=\left(p_{0}, \infty\right]$ and $P_{w}=\left[p_{0}, \infty\right]$. We consider the cases $p_{0}=1$ and $p_{0}>1$ separately, similarly as it was done in Section 2 .

By $\widetilde{\mathbb{Y}}_{1}$ we denote the second generation space $\left(Y_{\tau}, \rho, \mu\right)$ with construction based on $\tau_{n}=n$ and $F(n, i)=2^{i}$. Recall that $\mu$ is non-doubling.

Proposition 6. Let $\widetilde{\mathbb{Y}}_{1}$ be the metric measure space defined above. Then the associated centered operator $M^{c}$ is of strong type $(1,1)$, while the non-centered $M$ is of weak type $(1,1)$, but is not of strong type $(1,1)$.

Proof: First note that it is easy to verify that $M^{c}$ is of strong type $(1,1)$, by using exactly the same argument as in the proof of Proposition 5 . In the next step we show that $M$ is not of strong type $(1,1)$. Indeed, let $f_{n}=\delta_{y_{n}}, n \geq 1$. Then $\left\|f_{n}\right\|_{1}=d_{n}$ and for $i=1, \ldots, n$ we have $M f_{n}\left(y_{n i}^{\prime}\right) \geq\left(1+1 / n+2^{i}\right)^{-1}>1 / 2^{i+1}$ and hence we obtain $\left\|M f_{n}\right\|_{1} \geq$ $\sum_{i=1}^{n} 2^{i} d_{n} / 2^{i+1}=n\left\|f_{n}\right\|_{1} / 2$.

To complete the proof, it suffices to show that $M$ is of weak type $(1,1)$. Let $f \in \ell^{1}\left(\widetilde{\mathbb{Y}}_{1}\right), f \geq 0$, and consider $\lambda>0$ such that $E_{\lambda}(M f) \neq \emptyset$. If $\lambda<A_{Y_{\tau}}(f)$, then $\lambda\left|E_{\lambda}(M f)\right| /\|f\|_{1}<1$ follows. Therefore, from now on assume that $\lambda \geq A_{Y_{\tau}}(f)$. With this assumption, if for some $y \in T_{n}$ we have $M f(y)>\lambda$, then any ball $B$ containing $y$ and realizing $A_{B}(f)>\lambda$ must be a subset of $T_{n}$. Take any $n \in \mathbb{N}$ such that $E_{\lambda}(M f) \cap T_{n} \neq \emptyset$. If $\lambda<A_{T_{n}}(f)$, then

$$
\frac{\lambda\left|E_{\lambda}(M f) \cap T_{n}\right|}{\sum_{y \in T_{n}} f(y)|\{y\}|} \leq 2 .
$$


Assume that $\lambda \geq A_{T_{n}}(f)$ and take $y \in E_{\lambda}(M f) \cap T_{n}$. Now, any ball $B$ containing $y$ and realizing $A_{B}(f)>\lambda$ must be a proper subset of $T_{n}$. First, consider the case $E_{\lambda}(M f) \cap T_{n}^{\prime}=\emptyset$. If $y_{n} \in E_{\lambda}(M f) \cap T_{n}$, then we obtain $\sum_{y \in T_{n} \backslash T_{n}^{\prime}} f(y)|\{y\}|>\lambda\left|\left\{y_{n}\right\}\right|$ and since $\left|E_{\lambda}(M f) \cap T_{n}\right| \leq 2\left|\left\{y_{n}\right\}\right|$, (5) follows. Otherwise, if $y_{n} \notin E_{\lambda}(M f) \cap T_{n}$, then, necessarily, $f(y)>\lambda$ for every $y \in E_{\lambda}(M f) \cap T_{n}$ and hence (5) again follows. Finally, in the case $E_{\lambda}(M f) \cap T_{n}^{\prime} \neq \emptyset$, denote $j=\max \left\{i \in\{1, \ldots, n\}: M f\left(y_{n i}^{\prime}\right)>\right.$ $\lambda\}$. Therefore, $\sum_{y \in T_{n}} f(y)|\{y\}|>\lambda\left|\left\{y_{n j}^{\prime}\right\}\right|$ and combining this with the estimate $\left|E_{\lambda}(M f) \cap T_{n}\right| \leq 2\left|\left\{y_{n j}^{\prime}\right\}\right|$, we conclude that (5) follows. Since $\lambda\left|E_{\lambda}(M f) \cap T_{n}\right| / \sum_{y \in T_{n}} f(y)|\{y\}| \leq 2$ for any branch $T_{n}$ such that $E_{\lambda}(M f) \cap T_{n} \neq \emptyset$, we have $\lambda\left|E_{\lambda}(M f)\right| /\|f\|_{1} \leq 2$ and, consequently, $\|M f\|_{1, \infty} \leq 2\|f\|_{1}$.

Now, fix $p_{0} \in(1, \infty)$ and consider $\widetilde{\mathbb{Y}}_{p_{0}}=\left(Y_{\tau}, \rho, \mu\right)$ with construction based on $\tau_{n}=\tau_{n}\left(p_{0}\right)$ and $F(n, i)=F_{p_{0}}(n, i)$, defined in the same way as in Subsection 2.2 , by using the auxiliary sequences $c_{n}, e_{n}$, and $m_{n j}$, $s_{n j}, j \in\left\{1, \ldots, e_{n}\right\}, n \in \mathbb{N}$.

Proposition 7. Let $\widetilde{\mathbb{Y}}_{p_{0}}$ be the metric measure space defined above. Then the associated centered maximal operator $M^{c}$ is of strong type $(1,1)$, while the non-centered $M$ is of weak type $\left(p_{0}, p_{0}\right)$, but is not of strong type $\left(p_{0}, p_{0}\right)$.

Proof: First note once again that it is easy to verify that $M^{c}$ is of strong type $(1,1)$, by using the same argument as in the proof of Proposition 5. In the next step we show that $M$ is not of strong type $\left(p_{0}, p_{0}\right)$. Indeed, let $f_{n}=\delta_{x_{n}}, n \geq 1$. Then $\left\|f_{n}\right\|_{p_{0}}^{p_{0}}=d_{n}$ and for $i=1, \ldots, \tau_{n}$ we have $M f_{n}\left(y_{n i}^{\prime}\right) \geq\left(1+1 / \tau_{n}+m_{n j(n, i)}\right)^{-1} \geq\left(2\left(1+m_{n j(n, i)}\right)\right)^{-1}$ and hence

$$
\begin{aligned}
\left\|M f_{n}\right\|_{p_{0}}^{p_{0}} & \geq \sum_{j=1}^{e_{n}} \sum_{k=1}^{s_{n j}} \frac{d_{n} m_{n j}}{\left(2\left(1+m_{n j}\right)\right)^{p_{0}}}=d_{n} \sum_{j=1}^{e_{n}} \frac{s_{n j} m_{n j}}{\left(2\left(1+m_{n j}\right)\right)^{p_{0}}} \\
& \geq d_{n} \sum_{j=1}^{e_{n}} \frac{2^{1-j-p_{0}} n c_{n}}{\left(1+m_{n j}\right)^{p_{0}}}=2^{-p_{0}} d_{n} \sum_{j=1}^{e_{n}} \frac{n c_{n}}{(1+n)^{p_{0}}} \\
& =2^{-p_{0}} e_{n} \frac{n c_{n}}{(1+n)^{p_{0}}}\left\|f_{n}\right\|_{p_{0}}^{p_{0}} .
\end{aligned}
$$

Since $\lim _{n \rightarrow \infty} e_{n}=\infty$ and $\lim _{n \rightarrow \infty} \frac{n c_{n}}{(1+n)^{p_{0}}}=1$, we are done.

To complete the proof, it suffices to show that $M$ is of weak type $\left(p_{0}, p_{0}\right)$. Let $f \in \ell^{p_{0}}\left(\widetilde{\mathbb{Y}}_{p_{0}}\right), f \geq 0$, and consider $\lambda>0$ such that $E_{\lambda}(M f) \neq \emptyset$. If $\lambda<A_{Y_{\tau}}(f)$, then using the inequality $\|f\|_{1} \leq$ 
$\|f\|_{p_{0}}\left|Y_{\tau}\right|^{1 / q_{0}}$, we obtain $\lambda^{p_{0}}\left|E_{\lambda}(M f)\right| /\|f\|_{p_{0}}^{p_{0}}<1$. Therefore, from now on assume that $\lambda \geq A_{Y_{\tau}}(f)$. Take any $n \in \mathbb{N}$ such that $E_{\lambda}(M f) \cap T_{n} \neq \emptyset$. If $\lambda<A_{T_{n}}(f)$, then using similar argument as above we have

$$
\frac{\lambda^{p_{0}}\left|E_{\lambda}(M f) \cap T_{n}\right|}{\sum_{y \in T_{n}} f(y)^{p_{0}}|\{y\}|} \leq 1
$$

Consider $\lambda \geq A_{T_{n}}(f)$. Assume that $E_{\lambda}(M f) \cap T_{n}^{\prime}=\emptyset$. If $\lambda<A_{T_{n} \backslash T_{n}^{\prime}}(f)$, then (6) again follows. Otherwise, if $\lambda \geq A_{T_{n} \backslash T_{n}^{\prime}}(f)$, then we consider two cases. If $y_{n} \in E_{\lambda}(M f)$, then we obtain $f\left(y_{n}\right) \geq \lambda$ and hence

$$
\frac{\lambda^{p_{0}}\left|E_{\lambda}(M f) \cap T_{n}\right|}{\sum_{y \in T_{n}} f(y)^{p_{0}}|\{y\}|} \leq \frac{2 \lambda^{p_{0}}\left|\left\{y_{n}\right\}\right|}{\sum_{y \in T_{n}} f(y)^{p_{0}}|\{y\}|} \leq 2
$$

In the other case, if $y_{n} \notin E_{\lambda}(M f)$, then $f(y)>\lambda$ holds for every $y \in$ $E_{\lambda}(M f) \cap T_{n}$ and hence (6) follows one more time. Now assume that $E_{\lambda}(M f) \cap T_{n}^{\prime} \neq \emptyset$. See that $\left|E_{\lambda}(M f) \cap T_{n}\right| \leq 3\left|E_{\lambda}(M f) \cap T_{n}^{\prime}\right|$. Consider the case $f\left(y_{n}\right)<\left(1+1 / \tau_{n}+m_{n e_{n}}\right) \lambda / 3$. If $y_{n i}^{\prime} \in E_{\lambda}(M f) \cap T_{n}^{\prime}$ for some $i \in\left\{1, \ldots, \tau_{n}\right\}$, then $f\left(y_{n i}^{\prime}\right) \geq \lambda / 3$ or $f\left(y_{n i}\right)\left|\left\{y_{n i}\right\}\right| \geq\left|\left\{y_{n i}^{\prime}\right\}\right| \lambda / 3$ and hence $f\left(y_{n i}^{\prime}\right)^{p_{0}}\left|\left\{y_{n i}^{\prime}\right\}\right|+f\left(y_{n i}\right)^{p_{0}}\left|\left\{y_{n i}\right\}\right| \geq\left|\left\{y_{n i}^{\prime}\right\}\right|(\lambda / 3)^{p_{0}}$, which implies

$$
\frac{\lambda^{p_{0}}\left|E_{\lambda}(M f) \cap T_{n}\right|}{\sum_{y \in T_{n}} f(y)^{p_{0}}|\{y\}|} \leq \frac{3 \lambda^{p_{0}}\left|E_{\lambda}(M f) \cap T_{n}^{\prime}\right|}{\sum_{y \in T_{n}} f(y)^{p_{0}}|\{y\}|} \leq 3^{p_{0}+1} .
$$

Finally, in the case $f\left(y_{n}\right) \geq\left(1+1 / \tau_{n}+m_{n e_{n}}\right) \lambda / 3$, denote $r=\min \{j \in$ $\left.\left\{1, \ldots, e_{n}\right\}: f\left(y_{n}\right) \geq \frac{\left(1+1 / \tau_{n}+m_{n j}\right) \lambda}{3}\right\}$. Let $T_{n}^{(r)}=\left\{y_{n i}^{\prime}: i \in\left\{1, \ldots, \sum_{j=1}^{r-1} s_{n j}\right\}\right\}$. Note that the case $T_{n}^{(r)}=\emptyset$ is possible. Assume that $T_{n}^{(r)} \neq \emptyset$. If $y_{n i}^{\prime} \in$ $E_{\lambda}(M f) \cap T_{n}^{(r)}$, then $f\left(y_{n i}^{\prime}\right)^{p_{0}}\left|\left\{y_{n i}^{\prime}\right\}\right|+f\left(y_{n i}\right)^{p_{0}}\left|\left\{y_{n i}\right\}\right| \geq\left|\left\{y_{n i}^{\prime}\right\}\right|(\lambda / 3)^{p_{0}}$ and hence

$$
\frac{\lambda^{p_{0}}\left|E_{\lambda}(M f) \cap T_{n}^{(r)}\right|}{\sum_{i: y_{n i}^{\prime} \in T_{n}^{(r)}}\left(f\left(y_{n i}^{\prime}\right)^{p_{0}}\left|\left\{y_{n i}^{\prime}\right\}\right|+f\left(y_{n i}\right)^{p_{0}}\left|\left\{y_{n i}\right\}\right|\right)} \leq 3^{p_{0}+1} .
$$


Moreover, we have

$$
\begin{aligned}
\frac{\lambda^{p_{0}}\left|E_{\lambda}(M f) \cap\left(T_{n} \backslash T_{n}^{(r)}\right)\right|}{f\left(y_{n}\right)^{p_{0}}\left|\left\{y_{n}\right\}\right|} & \leq\left(\frac{3}{1+m_{n r}}\right)^{p_{0}} \frac{\left|T_{n} \backslash T_{n}^{(r)}\right|}{\left|\left\{y_{n}\right\}\right|} \\
& \leq\left(\frac{3}{1+m_{n r}}\right)^{p_{0}} \frac{\left.3 \mid\left(T_{n} \backslash T_{n}^{(r)}\right) \cap T_{n}^{\prime}\right) \mid}{\left|\left\{y_{n}\right\}\right|} \\
& \leq\left(\frac{3}{1+m_{n r}}\right)^{p_{0}} 3 \sum_{j=r}^{e_{n}} n c_{n} 2^{2-j} \\
& <2^{3-r} 3^{p_{0}+1} n c_{n}\left(\frac{1}{1+m_{n r}}\right)^{p_{0}} \\
& =4 \cdot 3^{p_{0}+1} \frac{n c_{n}}{(1+n)^{p_{0}}} \leq 4 \cdot 3^{p_{0}+1} .
\end{aligned}
$$

Therefore, regardless of the possibilities, $T_{n}^{(r)}=\emptyset$ or $T_{n}^{(r)} \neq \emptyset$, we obtain $\lambda^{p_{0}}\left|E_{\lambda}(M f) \cap T_{n}\right| / \sum_{y \in T_{n}} f(y)^{p_{0}}|\{y\}| \leq 4 \cdot 3^{p_{0}+1}$. Since $\lambda \mid E_{\lambda}(M f) \cap$ $T_{n}\left|/ \sum_{y \in T_{n}} f(y)^{p_{0}}\right|\{y\} \mid \leq 4 \cdot 3^{p_{0}+1}$ for any branch $T_{n}$ such that $E_{\lambda}(M f) \cap$ $T_{n} \neq \emptyset$, we have $\lambda^{p_{0}}\left|E_{\lambda}(M f)\right| /\|f\|_{p_{0}}^{p_{0}} \leq 4 \cdot 3^{p_{0}+1}$ and consequently $\|M f\|_{p_{0}, \infty}^{p_{0}} \leq 4 \cdot 3^{p_{0}+1}\|f\|_{p_{0}}^{p_{0}}$.

\section{Proof of Theorem 1}

All spaces discussed above were constructed in such a way as to avoid any interactions between the different branches in the context of considerations relating to the existence of the weak and strong type inequalities. Therefore we can construct a new space consisting of two types of branches, one borrowed from some first generation space and one from some second generation space, and to ensure that the operators $M^{c}$ and $M$ inherit a particular property of a particular space. We explain the construction of such a space in detail proving Theorem 1.

Proof of Theorem 1: We consider a few cases. If the equalities $P_{s}^{c}=P_{s}$ and $P_{w}^{c}=P_{w}$ are supposed to hold, then the expected space may be chosen to be a first generation space. If, in turn, we have $P_{s}^{c}=P_{w}^{c}=$ $[1, \infty]$, but $P_{s} \neq[1, \infty]$, then the expected space may be chosen to be a second generation space. Finally, in other cases we can find spaces $\mathbb{X}=\left(X, \rho_{X}, \mu_{X}\right)$ and $\mathbb{Y}=\left(Y, \rho_{Y}, \mu_{Y}\right)$, of first and second generation, respectively, for which

- $P_{s}^{c}(\mathbb{X})=P_{s}(\mathbb{X})=P_{s}^{c}$ and $P_{w}^{c}(\mathbb{X})=P_{w}(\mathbb{X})=P_{w}^{c}$,

- $P_{s}^{c}(\mathbb{Y})=P_{w}^{c}(\mathbb{Y})=[1, \infty], P_{s}(\mathbb{Y})=P_{s}$, and $P_{w}(\mathbb{Y})=P_{w}$. 
Using $\mathbb{X}$ and $\mathbb{Y}$ and assuming that $X \cap Y=\emptyset$ we construct the space $\mathbb{Z}=\left(Z, \rho_{Z}, \mu_{Z}\right)$ as follows. Denote $Z=X \cup Y$. We define the metric $\rho_{Z}$ on $Z$ by

$$
\rho_{Z}(x, y)= \begin{cases}\rho_{X}(x, y) & \text { if }\{x, y\} \subset X \\ \rho_{Y}(x, y) & \text { if }\{x, y\} \subset Y \\ 2 & \text { in the other case }\end{cases}
$$

and the measure $\mu_{Z}$ on $Z$ by

$$
\mu_{Z}(E)=\mu_{X}(E \cap X)+\mu_{Y}(E \cap Y), \quad E \subset Z .
$$

It is not hard to show that $\mathbb{Z}$ has the following properties

- $P_{s}^{c}(\mathbb{Z})=P_{s}^{c}(\mathbb{X}) \cap P_{s}^{c}(\mathbb{Y})=P_{s}^{c} \cap[1, \infty]=P_{s}^{c}$,

- $P_{s}(\mathbb{Z})=P_{s}(\mathbb{X}) \cap P_{s}(\mathbb{Y})=P_{s}^{c} \cap P_{s}=P_{s}$,

- $P_{w}^{c}(\mathbb{Z})=P_{w}^{c}(\mathbb{X}) \cap P_{w}^{c}(\mathbb{Y})=P_{w}^{c} \cap[1, \infty]=P_{w}^{c}$,

- $P_{w}(\mathbb{Z})=P_{w}(\mathbb{X}) \cap P_{w}(\mathbb{Y})=P_{w}^{c} \cap P_{w}=P_{w}$,

and therefore it may be chosen to be the expected space. Finally, it is not hard to see that $\mu_{Z}$ is non-doubling, since it is bounded and there is a ball $B$ in $Z$ with radius $r=1$ and $|B|<\epsilon$ for any arbitrarily small $\epsilon>0$.

\section{Acknowledgement}

This article was largely inspired by the suggestions of my supervisor Professor Krzysztof Stempak. I would like to thank him for insightful comments and continuous help during the preparation of the paper.

\section{References}

[1] J. M. Aldaz, An example on the maximal function associated to a nondoubling measure, Publ. Mat. 49(2) (2005), 453-458. DOI: 10.5565 /PUBLMAT_49205_ 10.

[2] H.-Q. LI, La fonction maximale de Hardy-Littlewood sur une classe d'espaces métriques mesurables, C. R. Math. Acad. Sci. Paris 338(1) (2004), 31-34. DOI: 10.1016/j.crma.2003.11.005.

[3] H.-Q. LI, La fonction maximale non centrée sur les variétés de type cuspidale, J. Funct. Anal. 229(1) (2005), 155-183. DOI: 10.1016/j. jfa.2005.01.008.

[4] H.-Q. Li, Les fonctions maximales de Hardy-Littlewood pour des mesures sur les variétés cuspidales, J. Math. Pures Appl. (9) 88(3) (2007), 261-275. DOI: 10.1016/j.matpur.2007.06.006. 
Faculty of Pure and Applied Mathematics

Wrocław University of Science and Technology

Wyb. Wyspiańskiego 27

50-370 Wrocław

Poland

E-mail address: Dariusz.Kosz@pwr.edu.pl

Primera versió rebuda el 15 de març de 2016, darrera versió rebuda el 12 de setembre de 2016 . 